\title{
Baicalein alleviates tubular-interstitial nephritis in vivo and in vitro by down-regulating NF-kB and MAPK pathways
}

\author{
Yan Chen ${ }^{1}$, Yu Zheng ${ }^{1}$, Zhihong Zhou ${ }^{1}$ and Jinjun Wang ${ }^{2}$ \\ ${ }^{1}$ Department of Nephrology, The Second Affiliated Hospital and Yuying Children's Hospital of Wenzhou Medical University, \\ Wenzhou, China \\ ${ }^{2}$ Department of Transplantation, The First Affiliated Hospital of Wenzhou Medical University, Wenzhou, China
}

\begin{abstract}
Tubular-interstitial nephritis (TIN) is characterized by tubular cell damage and inflammatory lesions of kidneys. Baicalein (BAI) is a flavonoid compound found in the roots of Scutellaria baicalensis Georgi. The present study was undertaken to explore the anti-inflammatory and anti-oxidative effects of BAI on TIN patients and a lipopolysaccharide (LPS)-induced TIN cell model. The expression levels of interleukin-6 (IL-6), IL-10, and tumor necrosis factor $\alpha$ in serum samples of TIN patients and culture supernatants of renal proximal tubular epithelial cells (RPTECs) were evaluated using enzyme-linked immunosorbent assay. Creatinine clearance was calculated using the Cockcroft-Gault equation. Activities of malondialdehyde, superoxide dismutase, and glutathione peroxidase were also determined. Viability and apoptosis of RPTECs were measured using MTT assay and Guava Nexin assay, respectively. QRT-PCR was performed to determine the expressions of Bax, Bcl-2, nuclear factor kappa B ( $\mid \kappa B \alpha)$, and p65. Protein levels of Bax, Bcl-2, IкB $\alpha, p 65$, c-Jun N-terminal kinase, extracellular regulated protein kinases, and p38 were analyzed using western blotting. We found that BAI reduced inflammation and oxidative stress in vivo and in vitro. Moreover, BAI alleviated the LPS-induced RPTECs viability inhibition and apoptosis enhancement, as well as nuclear factor kappa B (NF-kB), and mitogen-activated protein kinase (MAPK) activation. Phorbol ester, an activator of NF-KB, attenuated the effects of BAI on LPS-induced inflammatory cytokine expressions in RPTECs. In conclusion, BAI had anti-inflammatory and anti-oxidative effects on TIN patients and LPS-induced RPTECs by down-regulating NF-KB and MAPK pathways.
\end{abstract}

Key words: Tubular-interstitial nephritis; Baicalein; Lipopolysaccharide; Anti-inflammatory effects; NF-кB pathway; MAPK pathway

\section{Introduction}

Tubular-interstitial nephritis (TIN) is characterized by tubular cell damage and inflammatory lesions of kidneys (1). Epidemiology research indicates that TIN is one of the most common diseases of kidneys (2). Common clinical symptoms of TIN include edema, hypertension, and proteinuria (3). At present, only symptomatic treatment is available for TIN due to its unclear pathogenesis (4). More experimental and clinical research is needed to explore the pathogenesis of TIN. In addition, searching for novel and more effective therapies for TIN is also urgently needed.

Lipopolysaccharide (LPS) is a component of the outer membrane of gram-negative bacteria (5). A study demonstrated that LPS could activate inflammatory response and oxidative stress in vitro and in vivo (6). Cell-based TIN models induced by LPS treatment have been widely used for testing new anti-inflammatory medicines for TIN (7).
Baicalein (BAI) is a flavonoid compound found in the roots of Scutellaria baicalensis Georgi (8). Previous studies have reported that BAI exerts many pharmacological effects including antimicrobial, anti-inflammatory, antihypertensive, antispasmodic, and anticancer (9-12). For anti-inflammatory effects, Patwardhan et al. demonstrated that BAI could suppress mitogen-induced $\mathrm{T}$ cell activation, proliferation, and cytokines secretion by downregulating nuclear factor kappa $B(N F-\kappa B)$ transactivation (13). Fan et al. (14) proved that BAl exhibited antiinflammatory activity in LPS-stimulated RAW264.7 macrophages through estrogen receptor- and NF-кB-dependent pathways. Furthermore, Cai et al. (15) reported that BAI protected tissues against periodontitis in rats by suppressing expressions of cyclo-oxygenase 2 and inducible nitric oxide synthase. He et al. (16) indicated that BAI attenuated inflammatory responses in LPS-induced mastitis in

Correspondence: Jinjun Wang: <wangjinjun543@126.com> 
mice by inhibiting NF-kB and mitogen-activated protein kinase (MAPK) pathways. The above findings suggest that BAI has wide anti-inflammatory effects and imply that BAl can be a potential therapeutic medicine for inhibiting inflammatory injury in TIN.

Therefore, this study was aimed to explore the antiinflammatory and anti-oxidative effects of BAI on TIN patients and primary human RPTECs induced by LPS. The possible underlying mechanisms involved in the $\mathrm{NF}-\kappa \mathrm{B}$ and MAPK pathways were also investigated.

\section{Material and Methods}

\section{Sample collection}

Thirty patients (12 males and 18 females, 18 to 55 years old) diagnosed with TIN and fifteen healthy people ( 7 males and 8 females, 21 to 46 years old) were enrolled in this study between 2012 and 2014 at The Second Affiliated Hospital and Yuying Children's Hospital of Wenzhou Medical University. The present study was approved by the Ethics Committee of the institution (approval number: IACUC-14-013). Written informed consent was obtained from all patients and healthy people.

\section{Experimental treatment}

Patients were randomly allocated into either the TIN group or TIN + BAI group with fifteen patients in each group. Fifteen healthy people acted as the control group. Patients in the TIN group did not receive any treatment during the experiment and patients in TIN + BAI group received BAl capsules ( $80 \mathrm{mg}$, Xiuzheng Pharmaceutical Group, China) with $1.2 \mathrm{~g}$ each, 3 times per day orally for 1 month. Immediately after the experiment, serum samples of all patients and healthy people were collected to measure the levels of interleukin (IL)-6, IL-10, and tumor necrosis factor $\alpha$ (TNF- $\alpha)$, activities of malondialdehyde (MDA), superoxide dismutase (SOD), and glutathione peroxidase (GSH-PX), as well as creatinine clearance (CCr).

\section{Cell culture and treatment}

Primary human RPTECs were purchased from FMGBio (Sciencell, Shanghai, China), cultured in REBM/ REGM bullet kit (Biowhittaker Inc., USA) following the manufacturer's instruction and confirmed by staining using $\gamma$-glutamyl transferase (Thermo Fisher Scientific, USA), a selective marker residing in the brush border of human RPTECs (17). LPS (Sigma-Aldrich, USA) was diluted in endotoxin-free sterile phosphate-buffered saline (PBS, Sigma-Aldrich) according to the manufacturer's protocol. RPTECs were treated with $1 \mu \mathrm{g} / \mathrm{mL}$ LPS for $24 \mathrm{~h}$ to stimulate inflammatory injury in this research (18). For BAI treatment, $100 \mu \mathrm{M}$ BAl was added into the culture medium simultaneously with LPS exposure. Phorbol ester (PMA, Sigma-Aldrich) was used as the activator of NF$\kappa B$ pathway.

\section{Cytokine expression assay}

The levels of cytokines (IL-6, IL-10, and TNF- $\alpha$ ) in serum and in culture supernatant of RPTECs after relevant treatment were measured using enzyme-linked immunosorbent assay (ELISA) kits (R\&D Systems, USA) according to the manufacturer's instructions. RPTECs were plated into 24-well plates (Corning Inc., USA) and treated with $1 \mu \mathrm{g} / \mathrm{mL}$ LPS and/or $100 \mu \mathrm{M}$ BAl for $24 \mathrm{~h}$. After incubation, $100 \mu \mathrm{L}$ aliquot of culture medium supernatant from each well was collected with Transferpettor (Thermo Fisher Scientific).

\section{CCr analysis}

$\mathrm{CCr}\left(\mathrm{mL} \cdot \mathrm{s}^{-1} \cdot\left(\mathrm{m}^{2}\right)^{-1}\right)$ was calculated using the Cockcroft-Gault equation $(140-$ age in years $) \times$ body weight $(\mathrm{kg}) / 72 /$ serum creatinine $(\mathrm{Cr})(\mathrm{mg} / \mathrm{dL})$, and $\times 0.85$ if female) according to a previous study (19).

\section{MDA, SOD, and GSH-PX analysis}

The activity of MDA in serum and RPTECs after treatment was measured using OxiSelect ${ }^{\mathrm{TM}}$ TBARS Assay kit (Cell Biolabs, USA) according to the manufacturer's instruction. SOD activity in serum and RPTECs was evaluated by the adrenaline method according to Misra and Fridovich (20). GSH-PX activity in serum and RPTECs was measured by the method with Ellman's reagent of Sedlak and Lindsay (21), as modified by Little and O'Brien (22).

\section{Cell viability assay}

MTT assay was performed to measure cell viability. Briefly, human RPTECs were seeded into 96-well plates (Corning Incorporated, USA) with $10^{4}$ cells/well and treated with $1 \mu \mathrm{g} / \mathrm{mL}$ LPS and/or $100 \mu \mathrm{M}$ BAI for $24 \mathrm{~h}$. Then, $10 \mu \mathrm{L}$ MTT solution ( $5 \mathrm{mg} / \mathrm{mL}$ in PBS) was added into the each well of the plate followed by incubation for $4 \mathrm{~h}$ at $37^{\circ} \mathrm{C}$. After that, the supernatant of each well was removed and $150 \mu \mathrm{L}$ dimethyl sulfoxide (DMSO, Beyotime, China) was added into the each well. Samples were agitated on a shaker for $15 \mathrm{~min}$, and the absorbance of each well at $570 \mathrm{~nm}$ was recorded using a microplate reader (Dynatech Laboratories, USA). Cell viability (\%) was determined as percent viability of the control group, which was taken as $100 \%$.

\section{Cell apoptosis assay}

Apoptosis of RPTECs was determined using Guava Nexin assay after LPS and/or BAI treatment. Briefly, RPTECs were seeded into 24-well plates with $3 \times 10^{4}$ cells per well and treated with $1 \mu \mathrm{g} / \mathrm{mL}$ LPS and/or $100 \mu \mathrm{M} \mathrm{BAI}$ for $24 \mathrm{~h}$. Then, cells in each group were harvested and stained with $100 \mu \mathrm{L}$ kit solution for $25 \mathrm{~min}$ at $37^{\circ} \mathrm{C}$ in the dark. After that, Guava easyCyte 8HT (Millipore, USA) was performed to record cell apoptosis, and data was analyzed using FCS Express software (De Novo Software, USA). 
A

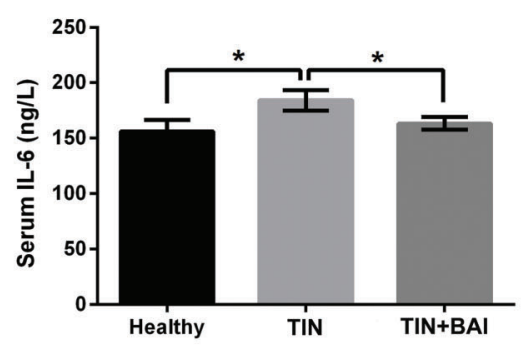

C

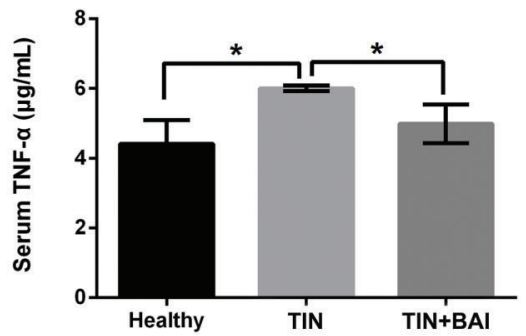

$\mathbf{E}$
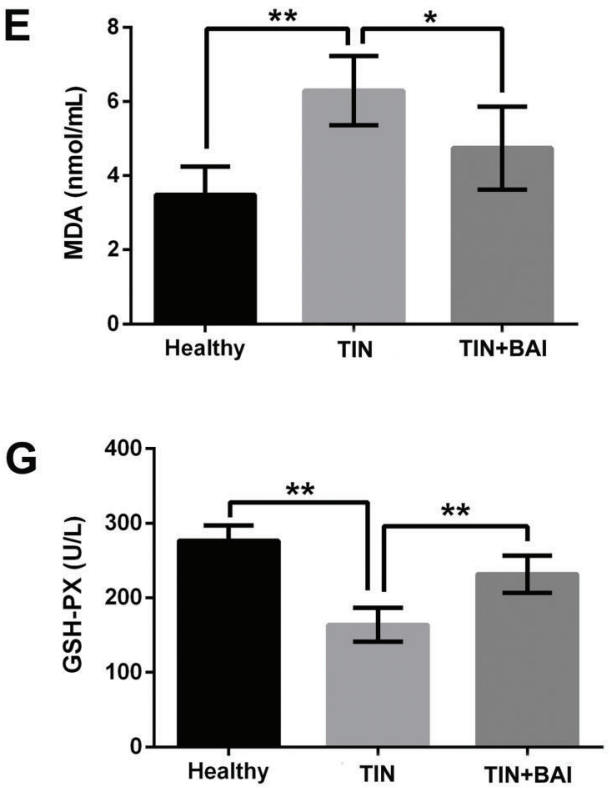

B

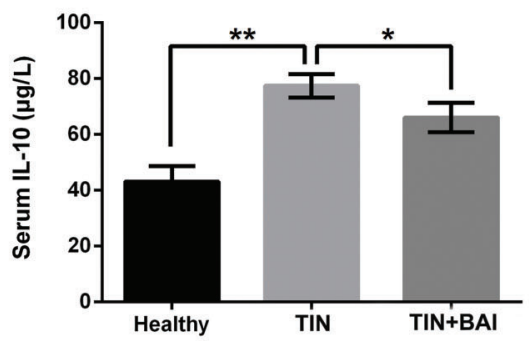

D

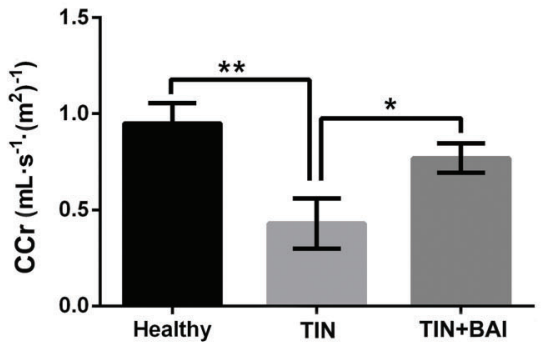

$\mathbf{F}$

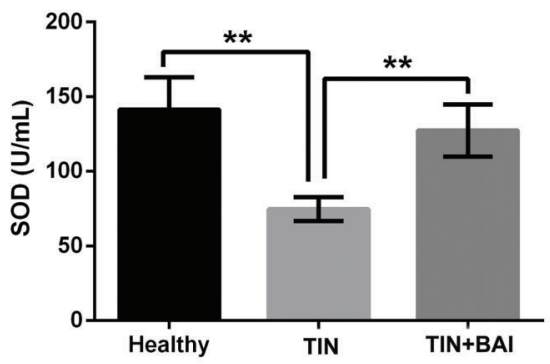

Figure 1. $A-C$, BAI treatment reduced the levels of IL-6, IL-10, and TNF- $\alpha$ in serum of TIN patients. $D$, BAI treatment enhanced the patient's CCr. E-G, BAI treatment reduced the activity of MDA and enhanced the activities of SOD and GSH-PX in serum of TIN patients. BAI: baicalein; TIN: tubular-interstitial nephritis; IL-6: interleukin-6; IL-10: interleukin-10; TNF- $\alpha$ : tumor necrosis factor- $\alpha$; CCr: creatinine clearance; MDA: malondialdehyde; SOD: superoxide dismutase; GSH-PX: glutathione peroxidase. Data are reported as means $\pm \mathrm{SE} .{ }^{*} \mathrm{P}<0.05,{ }^{* *} \mathrm{P}<0.01$ (ANOVA).

\section{Western blotting}

Briefly, the total proteins in RPTECs were isolated using RIPA lysis and extraction buffer (Thermo Fisher Scientific) and quantified using Pierce ${ }^{\mathrm{TM}}$ Rapid Gold BCA Protein Assay Kit (Thermo Fisher Scientific). Then, equal concentrations of protein samples were separated by electrophoresis on sodium dodecyl sulfate (SDS)-polyacrylamide gels and transferred onto polyvinylidene difluoride (PVDF) membranes (Millipore) with a semidry transfer system (Bio-Rad Laboratories, USA). After that, the membranes were blocked with $5 \%$ bovine serum albumin (BSA, Sigma-Aldrich) for $1 \mathrm{~h}$ at room temperature and incubated with primary antibodies overnight at $4^{\circ} \mathrm{C}$. All primary antibodies were prepared in $1 \%$ BSA solution with a dilution of 1:1000. After hybridization with primary antibodies, the membranes were washed three times with Tris-buffered 

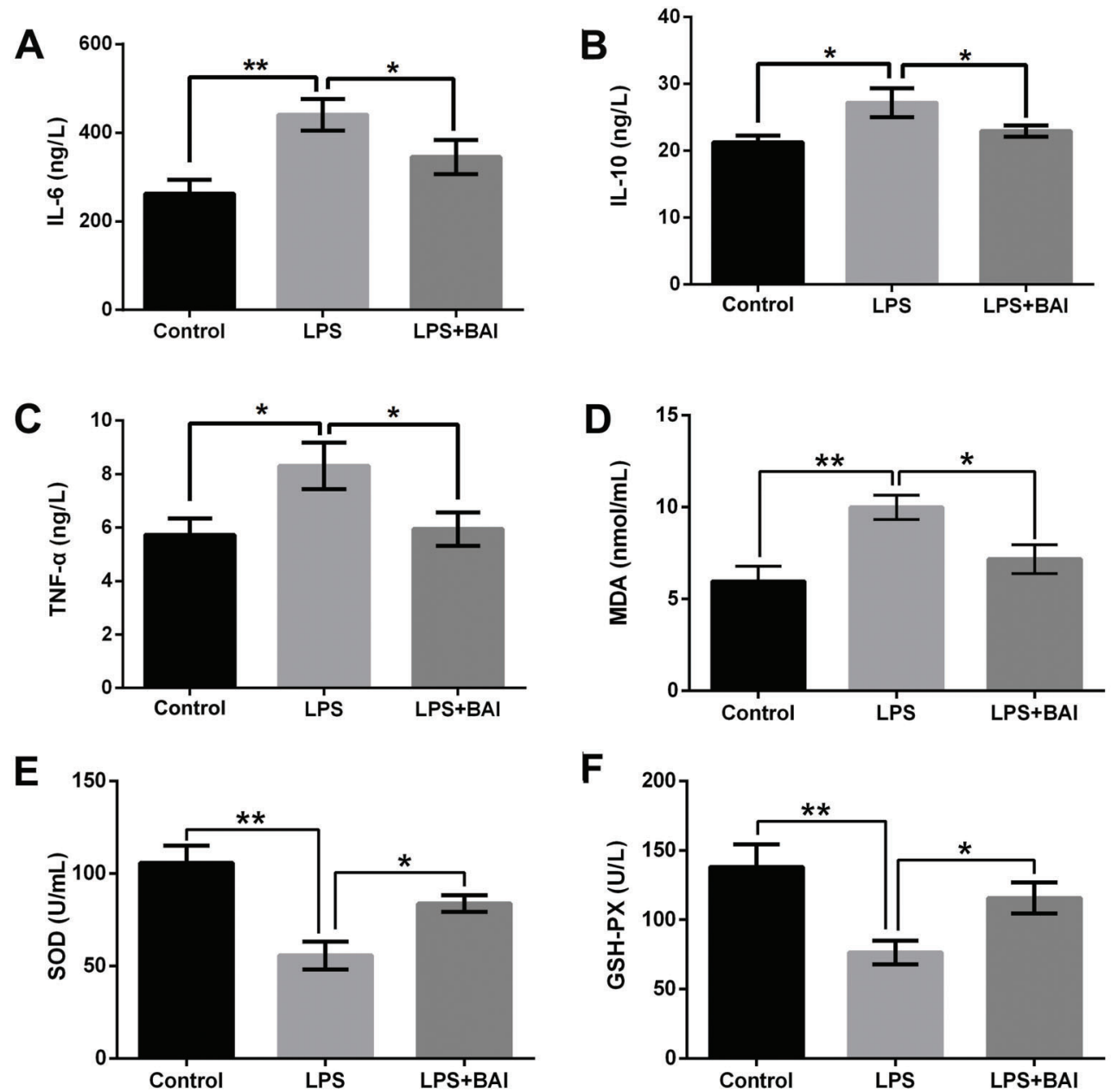

Figure 2. Effect of BAI in LPS-induced RPTECs in $A$, IL-6 expression; $B, \mathrm{IL}-10$ expression; $C$, TNF- $\alpha$ expression; $D$, MDA activity; E, SOD activity; F, GSH-PX activity. BAI: baicalein; LPS: lipopolysaccharide; RPTECs: renal proximal tubular epithelial cells; IL-6: interleukin-6; IL-10: interleukin-10; TNF- $\alpha$ : tumor necrosis factor- $\alpha$; MDA: malondialdehyde; SOD: superoxide dismutase; GSH-PX: glutathione peroxidase. Data are reported as means $\pm \mathrm{SE}$. ${ }^{*} \mathrm{P}<0.05$; ${ }^{* *} \mathrm{P}<0.01$ (ANOVA).

saline and Tween (TBST, Sigma-Aldrich) for $15 \mathrm{~min}$ and incubated with horseradish peroxidase-conjugated secondary antibodies (ab6721, ab6788, Abcam Biotechnology, USA) for $1 \mathrm{~h}$ at room temperature. After addition of western blotting Luminol reagents (Santa Cruz Biotechnology, USA), signals of proteins were recorded using Bio-Rad ChemiDoc $^{\text {TM }}$ XRS system (Bio-Rad Laboratories).

\section{Quantitative reverse transcriptase PCR (qRT-PCR)}

The total RNA in RPTECs after relevant treatment was isolated using RNeasy mini kit (Qiagen, USA) according to the manufacturer's instructions. cDNA was reversely transcribed using High Capacity cDNA Reverse Transcription kit (Applied Biosystems, USA). To detect the expressions of Bax, Bcl-2, IкB $\alpha$, and p65 in RPTECs, the Power SYBR-Green Master Mix Assay (Applied Biosystems) was used following the manufacturer's protocol. The conditions for the PCR program were set at $95^{\circ} \mathrm{C}$ for $15 \mathrm{~s}, 60^{\circ} \mathrm{C}$ for $30 \mathrm{~s}$, and $72^{\circ} \mathrm{C}$ for $45 \mathrm{~s}$ for 50 cycles). The data from the qRT-PCR were analyzed by CFX manager software (BioRad Laboratories). The expression of GAPDH acted as endogenous control in the respective samples.

\section{Statistical analysis}

All experiments in this research were performed in triplicate and repeated at least three times. The statistical analysis was performed using SPSS software (SPSS Inc., USA). The data of multiple experiments are reported as means $\pm \mathrm{SE}$. Statistical comparisons between two groups were performed using Student's $t$-test. One-way ANOVA followed by Tukey's multiple comparison test was used for comparisons involving three or more groups. The observations were considered significant with a $P$ value of $<0.05$. 
A

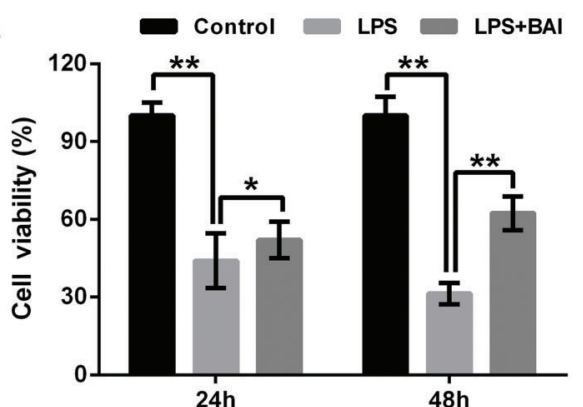

C

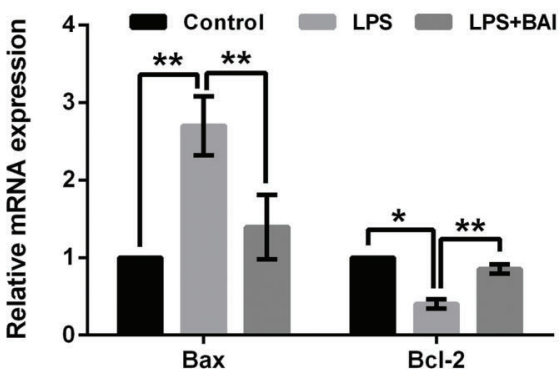

B

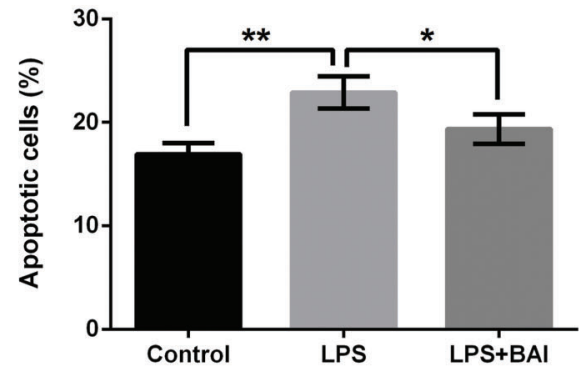

D

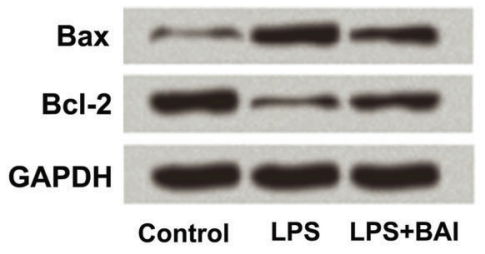

Figure 3. Effect of BAI on LPS-induced RPTECs. $A$, viability; $B$; apoptosis; $C$, mRNA expression of Bax and Bcl-2. $D$, Bax and Bcl-2 results by western blotting. BAI: baicalein; LPS: lipopolysaccharide; RPTECs: renal proximal tubular epithelial cells. Data are reported as means $\pm \mathrm{SE}$. ${ }^{*} \mathrm{P}<0.05 ;{ }^{* \star} \mathrm{P}<0.01$ (ANOVA).

\section{Results}

\section{$B A I$ reduced inflammatory injury and oxidative stress} of TIN patients

Figure $1 \mathrm{~A}$ and $\mathrm{C}$ shows that the levels of IL-6, IL-10, and TNF- $\alpha$ in serum samples of TIN patients with BAI treatment were significantly decreased $(P<0.05)$. Moreover, Figure 1D shows that BAI treatment remarkably enhanced $\mathrm{CCr}$ level $(\mathrm{P}<0.05)$. The activity of MDA in serum was significantly decreased and the activities of SOD and GSH$\mathrm{PX}$ in serum were increased after BAI treatment (Figure $1 E-G, P<0.05$ or $P<0.01)$. These results suggested that administration of BAI significantly decreased the inflammatory injury of TIN patients.

\section{BAI reduced LPS-induced inflammatory response and oxidative stress in RPTECs}

As shown in Figure 2A-C, LPS treatment dramatically increased the expression levels of IL-6, IL-10, and TNF- $\alpha$ in culture supernatant of RPTECs $(\mathrm{P}<0.05$ or $\mathrm{P}<0.01)$. $\mathrm{BAI}$ co-treatment significantly alleviated the LPS-induced increases of IL- 6 , IL-10, and TNF- $\alpha(P<0.05)$. In addition, the results of Figure 2D-F display that LPS treatment significantly enhanced the activity of MDA and reduced the activities of SOD and GSH-PX in RPTECs $(P<0.01)$. BAI co-treatment alleviated the LPS-induced MDA increase, as well as SOD and GSH-PX decreases in RPTECs $(P<0.05)$. These findings indicated that BAI attenuated LPS-induced inflammation and oxidative stress in RPTECs.

\section{BAI alleviated LPS-induced RPTECs viability} inhibition and apoptosis enhancement

Viability and apoptosis of RPTECs after LPS and/or BAI treatment were analyzed using MTT assay and Guava Nexin assay, respectively. Figure $3 \mathrm{~A}$ displays that LPS treatment significantly inhibited the viability of RPTECs in a timedependent manner $(P<0.01)$ and $B A I$ co-treatment remarkably alleviated the LPS-induced RPTECs viability inhibition $(P<0.05$ or $P<0.01)$. Figure 3B shows that BAl co-treatment noticeably attenuated the LPS-induced RPTECs apoptosis $(P<0.05)$. In addition, qRT-PCR showed that, compared to LPS alone, BAl co-treatment significantly decreased the mRNA expression of $\operatorname{Bax}(P<0.01)$ and increased the mRNA expression of $\mathrm{Bcl}-2$ in RPTECs (Figure $3 \mathrm{C}, \mathrm{P}<0.01$ ). Similar results were found in western blotting, which presented that BAI co-treatment attenuated the LPS-induced Bax protein level increase and Bcl-2 level decrease (Figure 3D). These findings indicated that BAI alleviated the LPS-induced RPTECs viability inhibition and apoptosis enhancement.

\section{BAl inhibited LPS-induced NF-кB and MAPK activation in RPTECs}

The mRNA and protein levels of $1 \kappa B \alpha$ and p65 in RPTECs after LPS and/or BAl treatment were analyzed by qRT-PCR and western blotting, respectively. Figure 4A and $B$ shows that LPS incubation enhanced the mRNA levels of $I \kappa B \alpha$ and $p 65$ in RPTECs $(P<0.01)$ and $B A I$ co-treatment significantly alleviated the LPS-induced $I_{\kappa} \mathrm{B} \alpha$ and p65 increases in RPTECs $(P<0.05)$. Similar results 
A

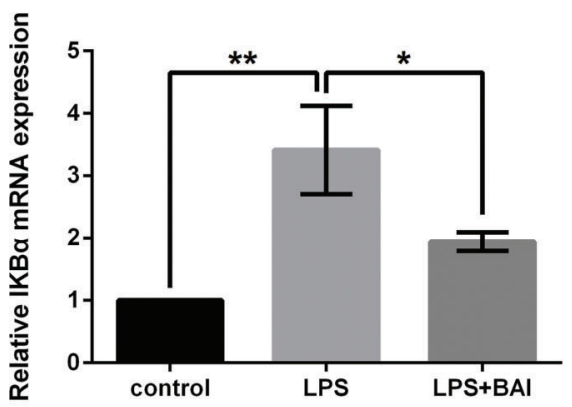

C

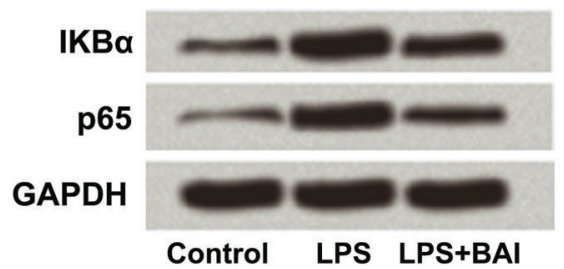

E

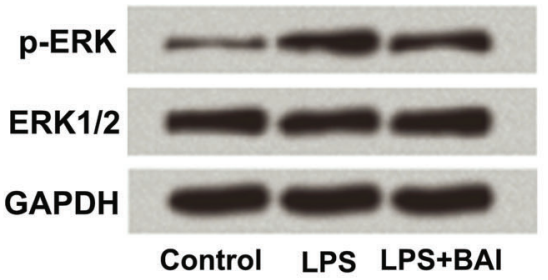

B

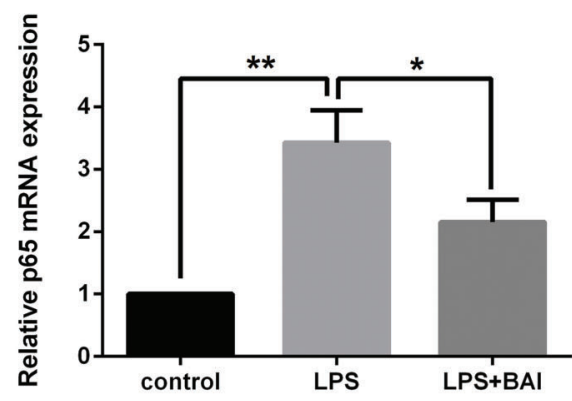

D

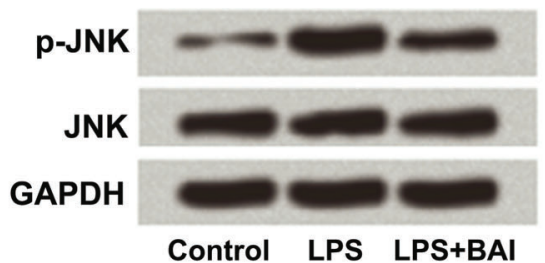

$\mathbf{F}$

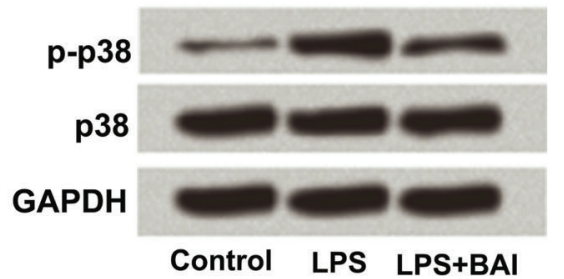

Figure 4. $A$ and $B$, Effect of BAI on LPS-induced mRNA expression of $\mathrm{I}_{\kappa} \mathrm{B} \alpha$ and p65 increase in RPTECs. $C$, Representative western blotting images of protein expressions of $1 \kappa \mathrm{B} \alpha$ and $\mathrm{p} 65 ; D-F$, protein expressions of $\mathrm{p}-\mathrm{JNK}$, $\mathrm{p}-\mathrm{ERK}$, and $\mathrm{p}-38$. BAI: baicalein; LPS: lipopolysaccharide; RPTECs: renal proximal tubular epithelial cells; NF-кB: nuclear factor kappa B; MAPK: mitogen-activated protein kinase; I $\mathrm{B} \alpha$ : inhibitor of $\mathrm{NF}-\kappa \mathrm{B}$; JNK: c-Jun $\mathrm{N}$-terminal kinase; ERK: extracellular regulated protein kinases. Data are reported as means $\pm \mathrm{SE} .{ }^{*} \mathrm{P}<0.05 ;{ }^{* *} \mathrm{P}<0.01$ (ANOVA).

were found in western blotting, which displayed that the protein levels of $1 \kappa B \alpha$ and p65 in RPTECs were also enhanced after single LPS incubation and reduced after LPS + BAI treatment (Figure 4C). Protein levels of the MAPK pathway were analyzed using western blotting. Figure 4D-F present that LPS incubation increased the protein levels of p-JNK, p-ERK, and p-p38 in RPTECs, whereas BAI co-treatment attenuated the effects of LPS on p-JNK, p-ERK1/2, and p-p38 in RPTECs. These findings suggested that $B A I$ inhibited the LPS-induced NF- $\mathrm{B}$ and MAPK pathways activation in RPTECs.

\section{BAl reduced LPS-induced inflammatory cytokine expressions in RPTECs by down-regulating NF-кB pathway}

To further confirm whether BAI reduces LPS-induced inflammatory cytokines in RPTECs by regulating NF- $\kappa B$ pathway, we administered the activator of NF- $\kappa B$ pathway PMA in our experiments (23). Then, the expression levels of IL-6, IL-10, and TNF- $\alpha$ were tested by ELISA. Results in
Figure $5 \mathrm{~A}-\mathrm{C}$ show that the expressions of IL-6, IL-10, and TNF- $\alpha$ were increased in LPS + BAI + PMA treatment group compared to LPS + BAI treatment group $(P<0.05)$. These findings suggested that incubation of PMA significantly attenuated the effects of BAI on LPS-induced inflammatory cytokine expressions in RPTECs.

\section{Discussion}

Inflammatory processes and oxidative stress are both associated with many diseases, including TIN (24-27). Chinese herbs have gained attention worldwide in the treatment of multiple diseases, including TIN (28). In this research, we found that $\mathrm{BAI}$, a flavonoid compound isolated from the roots of Scutellaria baicalensis, significantly reduced the inflammatory injury and oxidative stress of TIN patients, as well as LPS-induced RPTECs. Further results showed that BAI remarkably alleviated the LPS-induced RPTECs viability inhibition and apoptosis enhancement, as well as NF- $\kappa \mathrm{B}$ and MAPK activation. In addition, PMA attenuated 

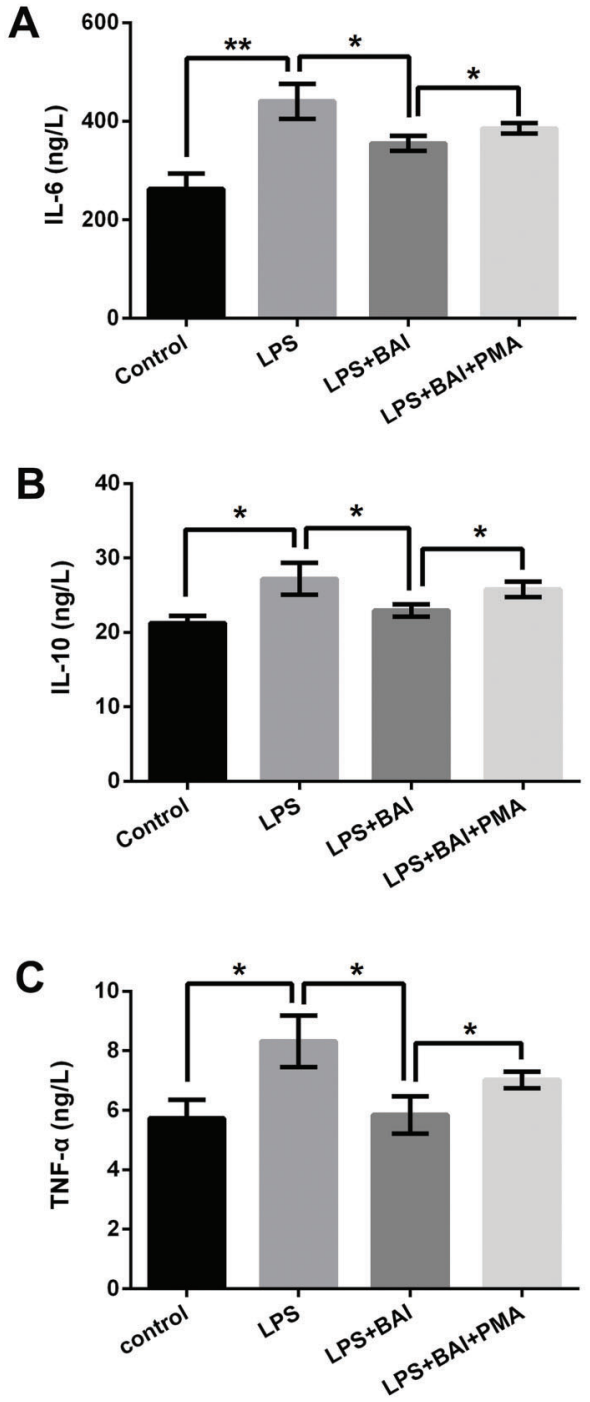

Figure 5. A, PMA alleviated the effects of BAI on LPS-induced IL-6 expression; $B$, IL-10 expression; and $C$, TNF- $\alpha$ expression in RPTECs. BAI: baicalein; LPS: lipopolysaccharide; RPTECs: renal proximal tubular epithelial cells; NF-кB: nuclear factor kappa B; PMA: phorbol ester; IL-6: interleukin-6; IL-10: interleukin-10; TNF- $\alpha$ : tumor necrosis factor- $\alpha$. Data are reported as means \pm SE. ${ }^{*} \mathrm{P}<0.05 ;{ }^{* *} \mathrm{P}<0.01$ (ANOVA).

the effects of BAI on LPS-induced inflammatory cytokine expressions, which implied that BAI reduced the LPSinduced RPTECs inflammatory injury and oxidative stress at least in part by down-regulating the NF- $\kappa B$ pathway.

BAI is a promising anti-inflammatory medicine due to its wide anti-inflammatory effects on multiple diseases $(24,26)$. Many previous studies demonstrated the antiinflammatory effects of BAI (13-16). Oxidative stress is often enhanced in patients with TIN, which promotes the expressions of inflammatory cytokines, such as IL-6, IL-10, and TNF- $\alpha$ (29). As an important indicator of renal function,
CCr is often decreased in patients with TIN (30). In this research, we found that the expression levels of IL-6, IL-10, and TNF- $\alpha$ were increased in serum samples of TIN patients and $\mathrm{CCr}$ was decreased. Moreover, the activity of MDA was increased and the activities of SOD and GSH-PX were decreased in serum samples of TIN patients. BAI treatment significantly reduced the expression levels of IL-6, IL-10, and TNF- $\alpha$ in serum samples, enhanced the patient's $\mathrm{CCr}$, and alleviated oxidative stress. Results in RPTECs showed that BAI treatment remarkably alleviated the LPS-induced IL-6, IL-10, and TNF- $\alpha$ expression increases, MDA activity increase, and SOD and GSH-PX activities decreases in RPTECs. These findings suggested that BAl exerted anti-inflammatory and anti-oxidative effects on TIN patients and the LPS-induced TIN cell model.

Excessive oxidative stress can promote the expression of inflammatory cytokines (31). Superabundant inflammatory cytokines production can damage mitochondrial function and then induce cell apoptosis (32). In this research, LPS treatment inhibited RPTECs cell viability and induced cell apoptosis. The expression of Bax was increased and the expression of $\mathrm{Bcl}-2$ was decreased in RPTECs after LPS treatment. BAI treatment effectively alleviated the LPS-induced cell viability inhibition and cell apoptosis enhancement. These results suggested that BAI also exerted anti-injury effects on the LPS-induced TIN cell model.

Previous studies demonstrated that BAI exerted antiinflammatory activity by suppressing NF- $\mathrm{B}$ a and MAPK pathways $(13,16)$. NF- $\kappa B$ and MAPK signaling pathways play critical regulatory roles in multiple cell functions including cell proliferation, apoptosis, autophagy, differentiation, and inflammation $(33,34)$. In terms of cell inflammatory response, NF- $\kappa \mathrm{B}$ could transfer to nuclear stimulating expressions of inflammatory cytokines (35). In our study, we found that LPS single treatment significantly up-regulated the expressions of $I_{\kappa} \mathrm{B} \alpha, \mathrm{p} 65, \mathrm{p}-\mathrm{JNK}, \mathrm{p}$-ERK, and p-p38 in RPTECs, and BAI co-treatment obviously alleviated these increases. Moreover, PMA, an activator of NF-KB (23), incubation remarkably alleviated the effects of BAI on LPSinduced inflammatory cytokine expressions. These results indicated that BAI exerted anti-inflammatory and anti-injury effects on the LPS-induced TIN cell model at least in part by inactivating NF- $\mathrm{NB}$ and MAPK signaling pathways.

To conclude, our research verified that BAI alleviated inflammatory injury in TIN patients and LPS-induced RPTECs by down-regulating NF- $\mathrm{KB}$ and MAPK pathways. This study explored the anti-inflammatory effects of BAl and provided a theoretical basis for its use in the treatment of TIN.

\section{Acknowledgments}

This work was supported by the Science and Technology Department of Wenzhou (Y20130199) and Zhejiang Provincial Natural Science Foundation of China (LQ13H 100003) as well as National Natural Science Foundation of China (81501382). 


\section{References}

1. Jeong HJ, Shin SJ, Lim BJ. Overview of IgG4-related tubulointerstitial nephritis and its mimickers. J PatholTransl Med 2016; 50: 26-36, doi: 10.4132/jptm.2015.11.09.

2. Gu X, Herrera GA. Light-chain-mediated acute tubular interstitial nephritis: a poorly recognized pattern of renal disease in patients with plasma cell dyscrasia. Arch Pathol Lab Med 2006; 130: 165-169.

3. Tomino Y, Kabuki K. Symptoms in patients with tubulointerstitial nephritis. Nihon rinsho. Japan J Clin Med 1995; 53: 1919-1924.

4. Tanaka T, Nangaku M. Pathogenesis of tubular interstitial nephritis. Contrib Nephrol 2011; 169: 297-310, doi: 10.1159/ 000314577.

5. Sperandeo P, Martorana AM, Polissi A. Lipopolysaccharide biogenesis and transport at the outer membrane of Gramnegative bacteria. Biochim Biophys Acta 2017; 1862: 14511460, doi: 10.1016/j.bbalip.2016.10.006.

6. Huang $\mathrm{X}$, Liu Y, Lu Y, Ma C. Anti-inflammatory effects of eugenol on lipopolysaccharide-induced inflammatory reaction in acute lung injury via regulating inflammation and redox status. Int Immunopharmacol 2015; 26: 265-271, doi: 10.1016/ j.intimp.2015.03.026.

7. Shui HA, Ka SM, Wu WM, Lin YF, Hou YC, Su LC, et al. LPS-evoked IL-18 expression in mesangial cells plays a role in accelerating lupus nephritis. Rheumatology (Oxford) 2007; 46: 1277-1284, doi: 10.1093/rheumatology/kem136.

8. Chen WP, Xiong Y, Hu PF, Bao JP, Wu LD. Baicalein inhibits MMPs expression via a MAPK-dependent mechanism in chondrocytes. Cell Physiol Biochem 2015; 36: 325-333, doi: 10.1159/000374075.

9. Sithisarn $P$, Nantateerapong $P$, Rojsanga $P$, Sithisarn $P$. Screening for antibacterial and antioxidant activities and phytochemical analysis of oroxylum indicum fruit extracts. Molecules 2016; 21: 446, doi: 10.3390/molecules21040446.

10. Lee W, Ku SK, Bae JS. Anti-inflammatory effects of Baicalin, Baicalein, and Wogonin in vitro and in vivo. Inflammation 2015; 38: 110-125, doi: 10.1007/s10753-014-0013-0.

11. Takizawa $\mathrm{H}$, DelliPizzi AMNasjletti A. Prostaglandin $\mathbf{I 2}$ contributes to the vasodepressor effect of baicalein in hypertensive rats. Hypertension 1998; 31: 866-871, doi: 10.1161/ 01.HYP.31.3.866

12. Cathcart MC, Useckaite Z, Drakeford C, Semik V, Lysaght J, Gately K, et al. Anti-cancer effects of baicalein in non-small cell lung cancer in vitro and in vivo. BMC Cancer 2016; 16 : 707, doi: 10.1186/s12885-016-2740-0.

13. Patwardhan RS, Sharma D, Thoh M, Checker R, Sandur SK. Baicalein exhibits anti-inflammatory effects via inhibition of NF-kappaB transactivation. Biochem Pharmacol 2016; 108: 75-89, doi: 10.1016/j.bcp.2016.03.013.

14. Fan GW, Zhang Y, Jiang X, Zhu Y, Wang B, Su L, et al. Antiinflammatory activity of baicalein in LPS-stimulated RAW264.7 macrophages via estrogen receptor and NF-kappaB-dependent pathways. Inflammation 2013; 36: 1584-1591, doi: 10.1007/ s10753-013-9703-2.

15. Cai X, Li C, Du G, Cao Z. Protective effects of baicalin on ligature-induced periodontitis in rats. J Periodontal Res 2008; 43: 14-21, doi: 10.1111/j.1600-0765.2007.00989.x.

16. He X, Wei Z, Zhou E, Chen L, Kou J, Wang J, et al. Baicalein attenuates inflammatory responses by suppressing TLR4 mediated NF-kappaB and MAPK signaling pathways in LPS-induced mastitis in mice. Int Immunopharmacol 2015; 28: 470-476, doi: 10.1016/j.intimp.2015.07.012.

17. Ding $\mathrm{Y}$, Yang $\mathrm{H}$, Xiang $\mathrm{W}, \mathrm{He} X$, Liao W, Yi Z. CD200R1 agonist attenuates LPS-induced inflammatory response in human renal proximal tubular epithelial cells by regulating TLR4-MyD88-TAK1-mediated NF-kappaB and MAPK pathway. Biochem Biophys Res Commun 2015; 460: 287-294, doi: 10.1016/j.bbrc.2015.03.026.

18. Ding Y, Liao W, He X, Xiang W, Lu Q. CSTMP Exerts antiinflammatory effects on LPS-induced human renal proximal tubular epithelial cells by inhibiting TLR4-mediated NF-kappaB pathways. Inflammation 2016; 39: 849-859, doi: 10.1007/ s10753-016-0315-5.

19. Katoh H, Nozue T, Asada T, Nakashima K, Kimura Y, Ito S, et al. Frequency and predictors of bleeding complications associated with anti-coagulant therapy using dabigatran in Japanese patients with atrial fibrillation. $A m$ J Cardiovasc Dis 2014; 4: 70-78.

20. Misra HP, Fridovich I. The role of superoxide anion in the autoxidation of epinephrine and a simple assay for superoxide dismutase. J Biol Chem 1972; 247: 3170-3175.

21. Sedlak J, Lindsay RH. Estimation of total, protein-bound, and nonprotein sulfhydryl groups in tissue with Ellman's reagent. Anal Biochem 1968; 25: 192-205, doi: 10.1016/ 0003-2697(68)90092-4.

22. Little C, O'Brien PJ. An intracellular GSH-peroxidase with a lipid peroxide substrate. Biochem Biophys Res Commun 1968; 31: 145-150, doi: 10.1016/0006-291X(68)90721-3.

23. Wang X, Wang Q, Hu W, Evers BM. Regulation of phorbol ester-mediated TRAF1 induction in human colon cancer cells through a PKC/RAF/ERK/NF-kappaB-dependent pathway. Oncogene 2004; 23: 1885-1895, doi: 10.1038/sj.onc. 1207312.

24. Basciano H, Federico L, Adeli K. Fructose, insulin resistance, and metabolic dyslipidemia. Nutri Metab (Lond) 2005; 2: 5, doi: 10.1186/1743-7075-2-5.

25. Esser N, Legrand-Poels S, Piette J, Scheen AJ, Paquot N. Inflammation as a link between obesity, metabolic syndrome and type 2 diabetes. Diabetes Res Clin Pract 2014; 105: 141-150, doi: 10.1016/j.diabres.2014.04.006.

26. Lastra G, Manrique C, McFarlane SI, Sowers JR. Cardiometabolic syndrome and chronic kidney disease. Curr Diab Rep 2006; 6: 207-212, doi: 10.1007/s11892-006-0036-5.

27. Schrier RW, Wang W, Poole BMitra A. Acute renal failure: definitions, diagnosis, pathogenesis, and therapy. The Journal of clinical investigation 2004; 114: 5-14, doi: 10.1172/ $\mathrm{JCl} 200422353$.

28. Ding D, Yan H, Zhen X. Effects of Chinese herbs in children with Henoch-Schonlein purpura nephritis: a randomized controlled trial. J Tradit Chin Med 2014; 34: 15-22, doi: 10.1016/S0254-6272(14)60048-0.

29. Sabry A, Elbasyouni SR, Sheashaa HA, Alhusseini AA, Mahmoud K, George SK, et al. Correlation between levels of TNF-alpha and IL-6 and hematological involvement in SLE Egyptian patients with lupus nephritis. Int Urol Nephrol 2006; 38: 731-737, doi: 10.1007/s11255-006-0047-9.

30. Bonnet F, Deprele C, Sassolas A, Moulin P, Alamartine E, Berthezene $F$, et al. Excessive body weight as a new 
independent risk factor for clinical and pathological progression in primary IgA nephritis. Am J Kid Dis 2001; 37: 720727, doi: 10.1016/S0272-6386(01)80120-7.

31. Kim YW, West $X Z$, Byzova TV. Inflammation and oxidative stress in angiogenesis and vascular disease. $J$ Mol Med (Berl) 2013; 91: 323-328.

32. Ahn YH, Yeon YB, Lee G, Mee KK, Kang SK. Molecular insights of the injured lesions of rat spinal cords: Inflammation, apoptosis, and cell survival. Biochem Biophysl Res Commun 2006; 348: 560-570, doi: 10.1016/j.bbrc.2006.07.105.
33. Hoesel B, Schmid JA. The complexity of NF-kappaB signaling in inflammation and cancer. Mol Cancer 2013; 12: 86, doi: 10.1186/1476-4598-12-86.

34. Su S, Duan J, Chen T, Huang X, Shang E, Yu L, et al. Frankincense and myrrh suppress inflammation via regulation of the metabolic profiling and the MAPK signaling pathway. Sci Rep 2015; 5: 13668, doi: 10.1038/srep13668.

35. Li Q, Verma IM. NF-kappaB regulation in the immune system. Nat Rev. Immunol 2002; 2: 725-734, doi: 10.1038/ nri910. 